\title{
Design Optimization and Demand Side Management of a Solar- Assisted Industrial Heating Using Agent-Based Modelling (ABM): Methodology and Case Study
}

\author{
Fitsum Bekele Tilahun ${ }^{1}$, Ramchandra Bhandari $^{1}$, and Menegesha $\mathrm{Mamo}^{2}$ \\ ${ }^{1}$ Renewable Energy Systems, ITT, TH Köln (Cologne University of Applied Science), Köln, Germany \\ ${ }^{2}$ Electrical \& Computer Engineering, Addis Ababa University Institute of Technology (AAIT), Addis Ababa, Ethiopia
}

\begin{abstract}
Heavy dependence on traditional fuels as well as connection to a significantly unreliable and inefficient grid (with about $20 \%$ energy loss) characterizes the Ethiopian industry. On the contrary, the country has many solar-suited industries for process heat augmentation in the low-to-medium temperature ranges. Thus, if properly designed and operated, solar thermal could give an opportunity for an efficient and cleaner alternative in these industries. This paper presents agent-based modeling (ABM) for an integrated optimization and demand side management (DSM) framework for solar-assisted industrial heating under varying load and weather condition. To demonstrate the validity and practicality of the proposed solution, a case study was conducted on Ethiopian textile industry. A payback period of 5.3 years and solar fraction of $66.7 \%$ was identified for an optimized system. Further with the implementation of DSM to guide the production policy of the industry, a 7.5\% and $8.4 \%$ improvement in payback and solar fraction was achieved.
\end{abstract}

\section{Introduction}

Global estimate for industrial energy use indicates that more than $66.7 \%$ of the total energy consumption is for process heat, out of which $50 \%$ is for low-to-medium temperature range [1]. According to the same study, about two-fifths of primary energy use in industries comes from natural gas or petroleum indicating a solar replication potential that is estimated to be $15 \mathrm{EJ}$ which would cover about $10 \%$ of industrial energy requirement by 2030 .

Identified promising industrial sectors for solar integration are: food, beverages, paper, textile, brick and blocks, chemical, plastic and flour by products [1-2]. Similar to other developing countries, these industrial sectors dominate the Ethiopian industry [3].

According to existing forecasts, the electricity consumption in Ethiopian industrial sector is expected to grow by approximately 9 folds of the 2015 consumption by 2030 [4]. However, these industries are connected to the significantly unreliable national grid [5]. Consequently, the supply disruptions during the production process result in huge loss to the industries. The said unreliability of the electricity supply in the country has compelled many industries to set up their own emergency backup systems and often the diesel generator is their preferred choice. The employment of alternative local energy resources (for example, the use of solar thermal technologies to deliver industrial heat requirement, etcetera) as well as demand side management (DSM) could solve this problem.

If properly designed and operated, renewable energy resources could give an opportunity for an efficient and cleaner alternative together with the aspired reliability as is in the power industry.

The work of this paper is an attempt to address three related issues that still need a research attention. First of all, the number and fragmented nature of solar promising industrial processes have resulted in a need for a sector specific solution [1-2, 6-12]. Then more importantly, gaining the benefits of these specific solutions requires a holistic optimization framework; an approach that considers the daily performance based on weather prediction, hot water consumption variation and temperature dynamics of the thermal system. Finally, investigating the performance reliability of these optimized solutions in the presence of uncertainties in energy generation and consumption is necessary. However, scarcely few studies have answered these research questions mainly due to the inability of existing tools to handle such task [13-15]. On the other hand, agentbased models, as derivatives of complexity science, has been shown to be well suited for a natural description of systems where there is a dynamic interaction to result in complex collective patterns [15]. The primary aim of this paper is to demonstrate the capability of agent-based hybrid models for optimization and DSM analysis of an industrial scale solar thermal system. These agents have continuous thermal and consumption models that are embedded and run in parallel. Data for modelling are from measurements taken at the chosen industry and some are from manufacturer's datasheet. An optimization experiment with the total annualized cost (operating and investment) was carried out to find the optimal collector 
area and storage volume. These optimal parameters are then exported back to the simulation environment to investigate the possibility for improved energy use by implementing DSM to guide the industry's production policy.

\section{Case study}

The investigated dyeing textile process case study is found at Bahir Dar textile factory, in northern Ethiopia $\left(11^{\circ} 36^{\prime} \mathrm{N}\right.$ $\left.37^{\circ} 24^{\prime} \mathrm{E}\right)$. The textile factory manufactures $100 \%$ cotton products, including yarns and fabrics. Electricity, diesel and furnace oil (FO) are the major energy source to the plant with annual consumptionn of 27.54 million $\mathrm{kWh}$, $166 \mathrm{~kL}$ and $615 \mathrm{~kL}$ respectively [17]. The textile plant has installed FO fired steam boiler for dying section. This boiler operates only during production of dying section and consumes an average of 250 litters FO per day running with efficiency below $70 \%$. Increase price and poor reliability of $\mathrm{FO}$ are the two main problems that challenge the dyeing section of the textile factory.
On the other hand, the textile site receives an average direct and diffused solar radiation of $6.8 \mathrm{kWh} / \mathrm{m}^{2}$ and $1.7 \mathrm{kWh} / \mathrm{m}^{2}[18]$.Thus it is easy to see that a solar thermal system would have a higher replication potential for replacing the existing inefficient FO based boiler for the dyeing section of the factory. The primary aim of this study is for optimal design of a solar thermal system for the dyeing textile process and demand side management (DSM) for further energy efficiency improvement opportunity. The dyeing machine parameter is depicted in Table 1.

\section{Methodology}

The structure of the implemented methodology is depicted in Fig -1. The implementation is modelled in a computational framework based on Anylogic 8.1 and embedded optimizer OptQuest. The proposed work is divided into four basic procedures: i) Agent definition and communication, ii) Optimization, iii) demand side management (DSM), and iv) performance evaluation.

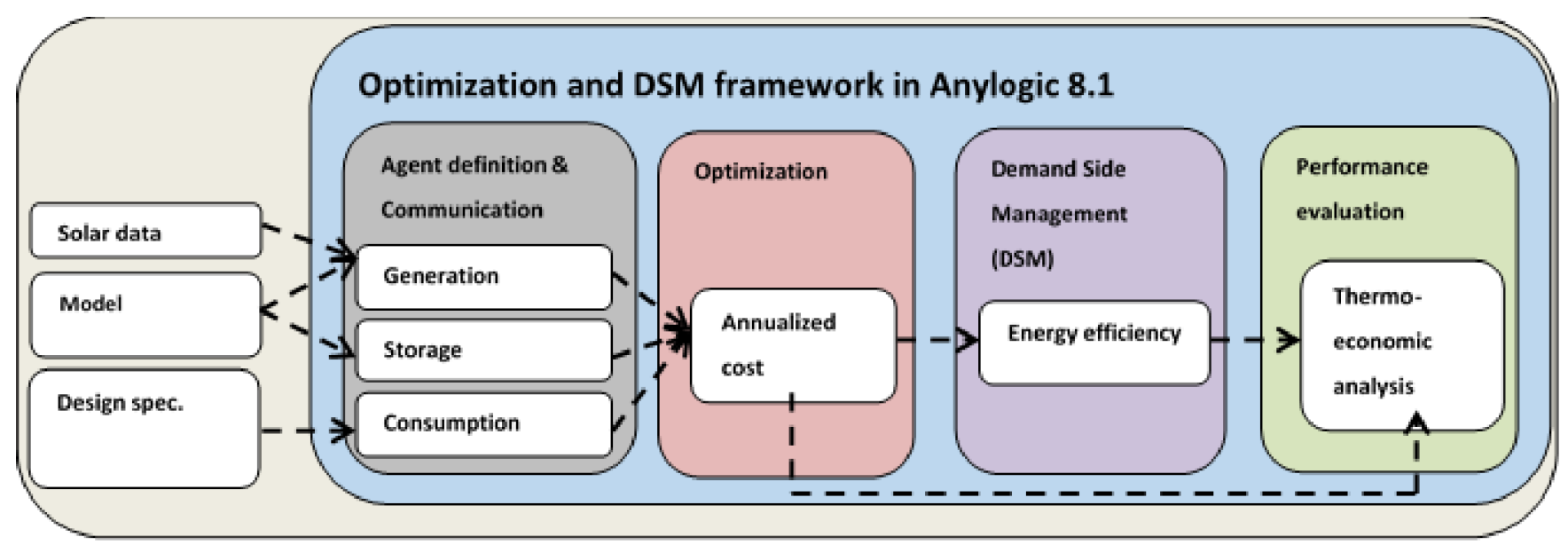

Fig. 1. The implemented framework.

\section{1 Agent definition and communication}

This task involves identifying which parts of the solarassisted industrial heating is important and creating these objects in the model as agents known as generation, storage, and consumption agents. The agent definition task creates these agents having a continuous thermal and/or demand models with embedded discrete events that run in parallel.

\subsubsection{Generation agent}

The generation agent used is Evacuated Tube Collector (ETC). ETC is the most efficient and convenient solar collector for low-to-medium temperature ranges [19]. The quasi-dynamic equation for ETC can be determined from $[20,21]$.

$$
\begin{aligned}
& Q(t)=\eta_{0} k_{\theta b}(\theta) I_{b}+\eta_{0} k_{\theta d}(\theta) I_{d}-a_{1}\left(T_{c o l l}-T_{a m b}\right)- \\
& a_{2}\left(T_{\text {coll }}-T_{a m b}\right)^{2}-c_{e f f} \frac{d T_{c o l l}}{d t}
\end{aligned}
$$

The collector parameters for the modeling of the generation agent are taken from [16].
Table 1. Dyeing textile machine parameters

\begin{tabular}{ll} 
Parameters & Values \\
\hline Mean daily production, $\mathrm{m}^{2}$ & 9,406 \\
Hot water cons, $\mathrm{L} / \mathrm{Kg}$ & $8-12$ \\
Process temp, ${ }^{\circ} \mathrm{C}$ & $40-60$ \\
Minimum fabric weight, $\mathrm{g} / \mathrm{m}^{2}$ & 265 \\
Maximum fabric width, $\mathrm{m}$ & 2.45 \\
Average washing machine speed, $\mathrm{m} / \mathrm{min}$ & 60 \\
Machine efficiency, $\%$ & 75 \\
\hline
\end{tabular}

\subsubsection{Storage agent}

As reported in [11] a stratified storage tank helps to store solar heat efficiently and ensure lowest possible inlet temperature to collector. Thus, the storage agent is modeled as $\mathrm{N}$ equally segmented storage tank layers with thermal dynamics, $\mathrm{T}_{\mathrm{n}}$, given as

$$
m_{n} c_{p} \frac{d T_{n}}{d t}=\dot{Q}_{c o n v, n}+\dot{Q}_{c o n d, n}-\dot{Q}_{\text {loss }, n}
$$


Where the convective, conductive, and loss stated in Eqn. (2) can be determined from temperature dynamics of neighboring segments $\left(T_{n-1}\right.$ and $\left.T_{n+1}\right)$ given by Eqns.(3-5).

$$
\begin{gathered}
\dot{Q}_{c o n v, n}=\dot{m}_{c} c_{p}\left(T_{n+1}-T_{n}\right)-\dot{m}_{w} c_{p}\left(T_{n}-T_{n-1}\right) \\
\dot{Q}_{c o n d, n}=\frac{A_{s t r, c} k}{d}\left(T_{n-1}+T_{n+1}-2 T_{n}\right) \\
\dot{Q}_{l o s s, n}=U A_{s t r, s}\left(T_{n}-T_{a m b}\right)
\end{gathered}
$$

This storage agent is composed of $\mathrm{N}$ segment chain where each segment's temperature state is derived from its own as well as the neighboring segments temperature dynamics. The first and last segment has the generation and consumption agent as the connecting Chain.

\subsubsection{Consumption agent}

The consumption agent has three states namely idle, not available, and processing. The idle states represents the waiting mode while the dyeing machine waits inputs from other textile processes proceeding it (desizing, bleaching, washing and drying). The not available state results when the dyeing machine is periodically maintained, occasionally breakdown or randomly stopped when the factory supply power is off. The final state i.e. processing state executes continuous dyeing in accordance input parameters listed in Table 1. While in this state, the thermal energy demand of the dyeing machine is updated in the agent. Once the input fabric is entirely processed, an event will be triggered to transit the dyeing machine to the idle state. The consumption agent estimates the dyeing machines availability and hence determines its thermal energy demand.

Once the agents are defined, the remaining step is to establish the agents' communication. This communication aims at smooth interaction among agents to make them able to react to external events such as thermal energy demand from consumption agent to storage agent. In order to accomplish this task, the agent communications generates and share these patterns as well as register their status

\subsection{Optimization}

The optimization tool used is OptQuest optimizer which is embedded into Anylogic 8.1. This optimizer is metaheuristics and is able to generalize under uncertainty during the optimization task.

An optimization experiment with total annual cost (annualized capital cost plus operating cost) of the thermal system is formulated. Both operating and investment cost for heat exchanger network, collector area, storage volume, including the control system and piping costs are considered in the objective function of the optimization experiment. The cost of investment for heat exchanger, Cex, is calculated using [21] as

$$
C_{e x}=e+f *\left(\frac{A_{t o t}}{N_{\text {min }}}\right)^{g} * N_{\text {min }}
$$

Whereas the cost for the collector and storage, $\mathrm{C}_{\mathrm{i}}$, can be approximated using the power low equation that relate the base size cost, $\mathrm{C}_{\mathrm{b}}$, and cost exponent factor, $\mathrm{s}$, given by Eqn. (7) [11].

$$
C_{i}=C_{b}\left(\frac{S_{i}}{S_{b}}\right)^{n}
$$

The annualized capital cost, $\mathrm{C}_{\mathrm{A}}$, of a new installation over a fixed period, $\mathrm{m}$, at a fixed rate of interest, $\mathrm{i}$, is given by

$$
C_{A}=C_{i} \frac{i(1+i)^{m}}{(1+i)^{m}-1}
$$

On the other hand, the operating cost, COP, is composed of the energy cost of auxiliary heat demand from boiler, $\mathrm{C}_{\mathrm{aux}}$, and maintenance $\operatorname{cost}, \mathrm{C}_{\text {main }}$, of the thermal system determined as

$$
\begin{gathered}
C_{O P}=C_{\text {aux }}+C_{\text {main }} \\
C_{\text {energy }}=C_{F O} \frac{Q_{F O}}{\eta_{b}}
\end{gathered}
$$

The cost of maintenance is assumed to be $1.5 \%$ of the fixed capital investment.

\subsection{Demand Side Management (DSM)}

The aim of DSM implementation is to reformulate the production policy of the dyeing textile process in order to use the available solar thermal energy efficiently. This is done by implementing a flexible production strategy that is in line with the seasonal and daily variation of the available solar energy. Thus, the DSM effort attempts to dynamically shift in time and/or vary the daily production rate. The allowable production rate is indirectly determined from the consumption agent that is described in section A.3. This DSM effort will result in efficient utilization of the generated solar energy thereby decreasing the associated loss.

\subsection{Performance Evaluation}

The optimal solar thermal configuration for the considered case study is determined at the optimization step as discussed in section 3.2. Afterwards the economic analysis, solar performance and carbon dioxide saving potential of the identified optimal configuration are calculated. The payback period, the total analyzed cost and the investment cost are indicators of the economic performance whereas solar gain and solar fraction points to the extent of solar energy utilization. On the other hand, the annual carbon dioxide emission reduction indicates the GHG mitigation potential of the proposed solar thermal system. The final step involves investigating the improvement in the aforementioned performance parameters when a DSM strategy is implemented.

\section{Results and discussion}

The first part of this section discusses the optimization result that gives the most economically feasible solution. Secondly, the demand side energy management is 
elaborated that leads to energy efficiency improvement potential in the considered dyeing textile process.

The optimization approach as discussed in section B was used to determine the most economical values for collector area and solar tank volume. The procedure was applied for ETC coupled to stratified storage tank with demand generated from the consumption agent.

The data shown in Table 2 gives an output of this optimization procedure.

Table 2. Summary of results.

\begin{tabular}{lll}
\hline & $\begin{array}{l}\text { Ref. } \\
\text { system }\end{array}$ & $\begin{array}{l}\text { With } \\
\text { DSM }\end{array}$ \\
\hline $\begin{array}{ll}\text { Optimized values } \\
\text { Collector area }\left(\mathrm{m}^{2}\right)\end{array}$ & 323 & 323 \\
Solar tank volume $\left(\mathrm{m}^{3}\right)$ & 18.1 & 18.1 \\
\hline Solar system performance & & \\
Solar heat gain $(\mathrm{MWh} / \mathrm{p})$ & 142.1 & 167.6 \\
Solar fraction $(\%)$ & 66.7 & 75 \\
\hline Economic analysis & & \\
Investment cost $(€)$ & 176838 & 176838 \\
Annualized total cost $(€ / \mathrm{p})$ & 52095 & 52095 \\
Payback period $(\mathrm{p})$ & 5.3 & 4.9 \\
\hline Saving & & \\
Fossil fuel $(\mathrm{MWh} / \mathrm{p})$ & 203.4 & 141.5 \\
Cost $(€ / \mathrm{p})$ & 9899 & 9830 \\
Co $(\mathrm{t} / \mathrm{p})$ & 52.0 & 47.0 \\
\hline
\end{tabular}

Before investigating DSM in the dyeing textile process, a flexible production policy needed to be formulated. This policy results from the knowledge of the dyeing machine's daily and monthly availability as estimated by the consumption agent. This consumption agent considers all stochastic events such as shortage of input fabric for the dyeing machine and represents them in probability distributions. The agent also takes deterministic events such periodic maintenance and represents them as occurrences with a given rate. The monthly dyeing machine availability is portrayed in Fig. 2.

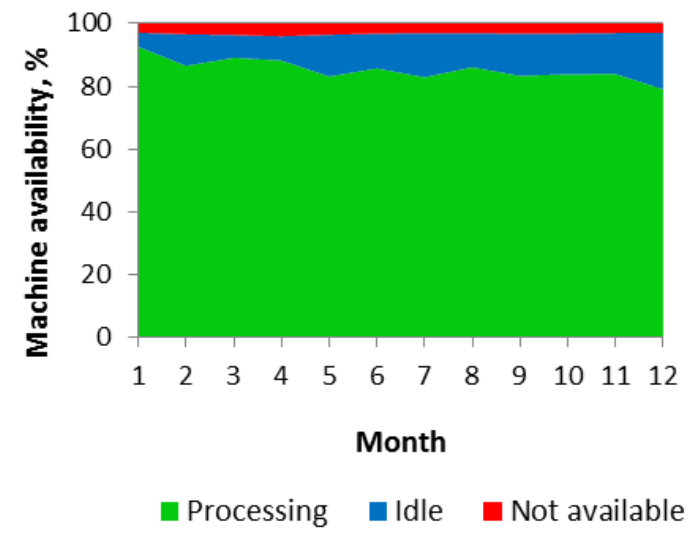

Fig. 2. Dyeing machine availability.

After estimating the machine's availability, the DSM procedure is implemented as follow: First, the consumption agent generates thermal demand based on the maximum daily dyeing machine's availability and tries to satisfy its demand from the storage agent (coupled to the generation agent). The excess of demand over supply is backlogged. On the succeeding days, the DSM procedure reviews the demand and supply inventory levels and decides on the daily production rate of the dyeing machine. This procedure goes on trying to match solar energy supply with production to arrive at a flexible production policy that results in maximum use of the generated solar energy. Thus, implementing the DSM strategy to guide the dyeing textile process, a maximum of $8.4 \%$ solar fraction with about $65.4 \mathrm{MWh}$ solar gain improvement could be achieved. Fig. 3 shows the monthly possible solar gain of the DSM implementation.

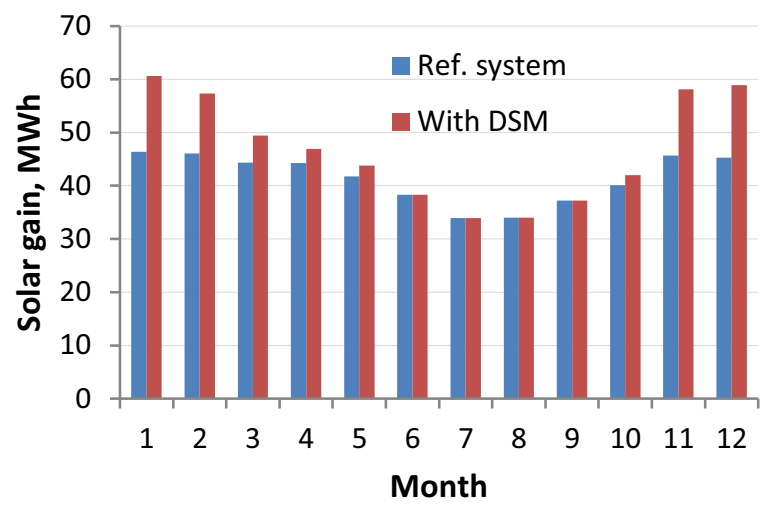

Fig. 3. DSM implementation for solar gain improvement.

\section{Conclusions}

The overall aim of this work was to arrive at an integrated optimization and demand side management approach, for decision support, in solar-assisted industrial heating. Demonstrated by a case study, it was found out that a payback period of 5.3 years and solar fraction of $66.7 \%$ was identified for an optimized system. With the implementation of DSM, to guide the production policy of the industry, a $7.5 \%$ and $8.4 \%$ improvement in payback and solar fraction was achieved.

\section{Acknowledgment}

The authors acknowledge the scholarship help from German Academic Exchange Service (DAAD) in supporting the $\mathrm{PhD}$ candidate.

\section{Nomenclature}

$\begin{array}{ll}A & \text { Area }\left(\mathrm{m}^{2}\right) \\ a_{1}, a_{2} & \text { Thermal loss coefficients }\left(\mathrm{W} /\left(\mathrm{m}^{2} \mathrm{~K}\right), \mathrm{W} /\left(\mathrm{m}^{2} \mathrm{~K}^{2}\right)\right) \\ e, f, g & \text { Fixed, scaling and, non-linear cost parameter } \\ C & \text { Cost }(€) \\ C_{F O} & \text { Specific cost of natural gas }(€ / \mathrm{J}) \\ c_{p} & \text { Specific heat capacity }(\mathrm{J} /(\mathrm{kg} \mathrm{K})) \\ c_{e f f} & \text { Effective thermal capacity } \mathrm{KJ} /\left(\mathrm{m}^{2} \mathrm{~K}\right) \\ I & \text { Solar irradiance }\left(\mathrm{W} / \mathrm{m}^{2}\right) \\ i & \text { Rate of interest } \\ k & \text { Thermal conductivity }(\mathrm{W} /(\mathrm{m} \mathrm{K}))\end{array}$




$\begin{array}{ll}k_{\theta b, k \theta d} & \text { Direct and diffused incidence angle modifier } \\ d & \text { Sstorage segment distance }(\mathrm{m}) \\ n & \text { Cost exponent factor } \\ N & \text { Number of heat exchanger } \\ \dot{m} & \text { Mass flow }(\mathrm{kg} / \mathrm{s}) \\ m & \text { Number of years } \\ Q & \text { Heat }(\mathrm{J}) \\ \dot{Q} & \text { Heat flow }(\mathrm{W}) \\ S & \text { Cost scaling factor } \\ T & \text { Temperature }\left({ }^{\circ} \mathrm{C}\right) \\ U & \text { Heat transfer coefficient }\left(\mathrm{W} /\left(\mathrm{m}^{2} \mathrm{~K}\right)\right)\end{array}$

\section{References}

1. Solar Heat for Industrial Processes, technology brief, International Renewable Energy Agency; January 2015.

2. Ashish K. Sharmaa, Chandan Sharmab, Subhash C. Mullicka, Tara C. Kandpala. Solar industrial process heating: A review. Renewable and Sustainable Energy Reviews, 2017; 78: 124-137

3. Growth and Transformation Plan II (GTP II) (2015/16-2019/20), national planning commission, the Federal Democratic Republic of Ethiopia; May 2016.

4. The Ethiopian power sector: a renewable future, Berlin Energy Transition Dialogue, Ministry of Water Irrigation and Electricity; March 2017

5. Study on the Energy Sector in Ethiopia, Embassy of Japan in Ethiopia, report available at http://www.et.emb-

japan.go.jp/electric_report_english.pdf. [Accessed 16.08.17].

6. Ashish K. Sharma, Chandan Sharma, Subhash C. Mullick, Tara C. Kandpal. Carbon mitigation potential of solar industrial process heating: paper industry in India. Cleaner Production, 2016; 112: 1683-1691

7. Mokhtar Ghazouani, Mohsine Bouya, Mohammed Benaissa. A new methodology to select the thermal solar collectors by localizations and applications. IEEE International Renewable and Sustainable Energy Conference (IRSEC), 2015:10-13.

8. M. Absi Halabi, A. Al-Qattan,n , A. Al-Otaibi. Application of solar energy in the oil industry-Current status and future prospects. Renewable and Sustainable Energy Reviews 2015; 43:296-314.

9. Badreddine Boutaghriout, Amina Bouakaz, and Chaâbane Hamouda. Investigation on the use of solar thermal energy in the agro food industry in Algeria. IEEE Renewable and Sustainable Energy Conference (IRSEC), 2013: 7-9.
10. Chaimaa El Mkadmi and Arifeen Wahed. Optimization of a solar thermal system for low temperature industrial heating process. IEEE Renewable and Sustainable Energy Conference (IRSEC), 2016: 14-17.

11. Tobias Eiholzera, Donald Olsena, Sebastian Hoffmanna, Barbara Sturmb, Beat Wellig. Integration of a solar thermal system in a medium-sized brewery using pinch analysis: methodology and case study. Applied Thermal Engineering, 2017; 113:1558-1568

12. Ilyes Ben Hassinea, Mariela Cotrado Sehgelmeble, Robert Söllb, Dirk Pietruschkaa. Control Optimization through Simulations of Large Scale Solar Plants for Industrial Heat Applications. Energy Procedia, 2015; 70: 595-604.

13. Krummenacher, P., Muster-Slawitsch, B. Methodologies and software tools for integrating solar heat into industrial processes. 13th International Conference on Sustainable Energy technologies, 2014.

14. Catherine S.E. Bale, Liz Varga, Timothy J. Foxon. Energy and complexity: New ways forward. Applied Energy, 2015; 138:150-159.

15. R. Pasupathy, S.-H. Kim, A. Tolk, R. Hill, and M. E. Kuhl. Multi-method modeling. Proceedings of the 2013 Winter Simulation Conference, 2013. P. 40894100.

16. The Solar Keymark. Database of certified products for collectors, http://www.solarkeymark.org/; 2017 [accessed 03.08.17].

17. Energy Audit Report of Bahir Dar Textile Share Company, Project Report No. 2013IB22, The Energy and Resources Institute, 2014

18. Measurement data from the WebelSolar(Grid) $3960 \mathrm{Wp}$ project, Bahir Dar university, Ethiopia, [Accessed 16.08.17].

19. M.A. Sabiha, R. Saidur, Saad Mekhilef, Omid Mahian. Progress and latest developments of evacuated tube solar collectors. Renewable and Sustainable Energy Reviews, 2015; 51:1038-1054.

20. Jie Deng, Xudong Yang, Pengsu Wang. Study on the second-order transfer function models for dynamic tests of flat-plate solar collectors Part I: A proposed new model and a fitting methodology. Solar Energy, 2015; 114:418-426.

21. Marcel Taal, Igor Bulatov, Jiri Klemes, and Petr Stehlik. Cost estimation and energy price forecasts for economic evaluation of retrofit projects. Applied Thermal Engineering, 23(14):1819-1835, 2003.

22. Vives, M., Energy production cost of solar thermal energy for industrial processes in spain. Catalonia institute for energy research (IREC), International conference on solar heating and cooling, June25-27, 2012. 